\title{
El caló español a comienzos del siglo XXI: el estado de la cuestión
}

\section{Spanish Gypsy at the Beginning of the 21st Century: the State of the Art}

\author{
ZuZANA KRINKovÁ [zuzana.krinkova@ff.cuni.cz] \\ Univerzita Karlova, República Checa \\ Ivo BuzEk [ibuzek@phil.muni.cz] \\ Masarykova univerzita, República Checa
}

\section{RESUMEN}

El objetivo del artículo es ofrecer una visión panorámica contextualizada sobre el caló español o hispanorromaní a comienzos del siglo XXI. En primer lugar, se presentará una breve introducción que ubicará el caló español en el contexto de las variedades del romaní en contacto con otras lenguas, los llamados pararromaníes, y se introducirán sus principales características que lo definen en el marco de la metodología de lenguas en contacto. A continuación, nos vamos a centrar en la recepción del caló en la sociedad mayoritaria española y cómo dicha recepción ha influido a la vez en la percepción y en la interpretación del término por parte del propio colectivo gitano. En esta parte trabajaremos tanto con los documentos producidos por personas ajenas al colectivo gitano, como con los formulados por los propios gitanos. Naturalmente, hasta bien entrado el siglo XX los testimonios proceden en general de los no-gitanos, mientras que los proferidos por los integrantes mismos del colectivo son más bien contemporáneos. No obstante, la atención principal se va a prestar al estado actual del caló español y, consecuentemente, a las estrategias de la política lingüística que intentan despertar su revitalización, con unos resultados que de momento son, como mínimo, poco fructuosos y poco convincentes.

\section{Palabras Clave}

Gitano español; pararromaní; sociolingüística; política lingüística; planificación lingüística

\section{Abstract}

The purpose of the article is to offer a contextualized panoramic view of the Spanish Gypsy or hispanorromani in the 21st century. In the first place, a brief introduction will be presented that will show the Spanish Gypsy in the context of other varieties of the Romani in contact with other languages, the so-called para-Romani, and the main characteristics that define it within the framework of languages in contact. In next step, we will focus on the reception of the Spanish Gypsy in the Spanish majority society and how the reception has influenced the perception and the interpretation of its notions by the Spanish Gypsy community. In this part we will work both with the documents produced by people who do not belong to the Gypsy community, as well as those formulated by the Spanish Gypsies themselves. Naturally, until the 20th century these testimonies come from non-Gypsies, but in last few decades there have appeared opinions from Spanish Gypsies as well. Nevertheless, the main attention is paid to the current status of the Spanish Gypsy language and, 
consequently, to the language policy strategies that try to awaken its revitalization. Unfortunately, the results are in the present moment neither much fruitful, nor convincing.

\title{
KEYWORDS
}

Spanish Gypsy; para-Romani; sociolinguistics; language policy; language planning

RECIBIDO 2019-10-10; ACEPTADO 2020-02-23

\begin{abstract}
Los autores agradecen a los revisores sus valiosos comentarios y sugerencias que han ayudado a mejorar considerablemente el texto. Los errores y omisiones que persisten son de nuestra propia responsabilidad.
\end{abstract}

\section{Diferenciación étnica del pueblo romaní y su nombre}

En la actualidad está generalmente aceptado que los romaníes provienen de la India. Las pruebas lingüísticas se inclinan más por un origen indio y por una consiguiente ola migratoria por el territorio de la India y fuera de él hacia occidente. El elemento persa y armenio en el vocabulario romaní también testimonian una estancia prolongada de los romaníes en el Oriente próximo antes de su llegada a Bizancio. Los registros históricos y el fuerte elemento griego en todos los dialectos romaníes muestran, además, que la presencia de los romaníes en Bizancio data a más tardar del siglo XI y que debió durar un largo período de tiempo, antes de que los romaníes se dispersaran por Europa, lo que ocurrió seguramente durante el siglo XIV. Los romaníes llegaron a España en el siglo XV (el primer documento es de 1425).

Los miembros del grupo étnico del pueblo romaní vivieron durante todo el tiempo dispersos o en mayores concentraciones entre una sociedad mayoritaria de la cual se diferenciaban antropológica, lingüística y socioculturalmente.

Los nombres propios de la etnia aparecen en doble forma (endónimos y exónimos). Los endónimos de los subgrupos romaníes se diferencian considerablemente. Los miembros de numerosos subgrupos romaníes se identifican con el nombre de rrom (rroma PL), 'hombre. La propia denominación étnica que utilizan algunos grupos, principalmente en la parte oriental y septentrional de Europa, puede considerarse también una forma conservadora de hacer referencia a la pertenencia étnica. Sirva de ejemplo la denominación sinti (cf. hindú sant 'unión', 'agrupación'1) en algunas partes de Europa occidental y oriental, además de manuš (rom. 'ser humano', por ejemplo, manouches en Francia), kale ('negros' PL, por ejemplo, calé en España y kaale en Finlandia), romaničel (de etimología poco clara, por ejemplo, Romany Chal en Gran Bretaña y errumantxela en el País Vasco). En algunas áreas, principalmente en los Balcanes y Europa oriental, predominan las denominaciones adoptadas de los idiomas del entorno, que están basadas en el modo tradicional de sustento de los diferentes grupos, por ejemplo, sepeči 'cesteros' (del turco sepetci).

1 Es una de las posibles explicaciones y es preciso tratarla con debida precaución, puesto que la flexión parece ser propia de un préstamo más reciente, lo que iría en contra de la hipótesis de su origen indio. 
Entre los exónimos la denominación más frecuente es la de cigano/cíngaro (cf. en alemán Zigeuner, en francés tsigane, en italiano zingaro, en portugués cigano; en España se utiliza a veces para denominar a los romaníes procedentes de los Balcanes). Este término surgió probablemente a partir del apodo athinganoi (atsínganoi), con el que en el Bizancio del siglo XI se hacía referencia a los herejes que practicaban la adivinación y la magia. Las mismas habilidades presentaban unos desconocidos romaníes que llegaron justo después de ellos y a los que se les trasladó esta denominación. Tanto el latín de la Edad Media como las lenguas de las diferentes naciones que entraron en contacto con los grupos romaníes adoptaron este nombre.

Otro exónimo común, el de egipcio (del que procede el inglés Gypsy, en español gitano), surgió seguramente como resultado del intercambio con el Pequeño Egipto en el Peloponeso griego, donde los romaníes vivieron un cierto período de tiempo. Los propios romaníes adoptaron en los inicios en la Edad Media el papel de peregrinos penitentes procedentes de Egipto.

En España se utilizaba también la expresión greciano, que probablemente tiene su origen en la ola migratoria de romaníes de finales del siglo XV que, al parecer, llegaron de Grecia huyendo de los turcos, mientras que en los territorios de la Corona de Aragón se utilizaba durante siglos la denominación catalana bomía 'bohemiano', relacionada con el antiguo término de francés bohémien para 'gitano' y documentada en forma de bohemiano también en español ${ }^{2}$. En la segunda mitad del siglo XIX, llegó a Europa una ola migratoria de romaníes valacos. Este es probablemente el origen de los húngaros españoles.

\section{El pararromaní}

El asentamiento en Europa de la población romaní es irregular y no constituye un continuo lingüístico en el propio sentido de la palabra. Mientras que en algunas zonas existe una gran densidad de población romaní y en el territorio de un Estado encontramos también una gran cantidad de grupos romaníes que se diferencian lingüística y culturalmente (por ejemplo, en los Balcanes o en la Europa central), otras áreas presentan una relativa homogeneidad y la concentración de la etnia romaní es menor (por ejemplo, en la Europa occidental). Algunos subgrupos romaníes, además, no hablan romaní.

Además de los dialectos romaníes flexivos, existen también variantes lingüísticas en las que se ha insertado en la gramática de las lenguas mayoritarias una cantidad considerable del léxico romaní (con restos de la flexión romaní). La ciencia actual que estudia el romaní se inclina al definir estas variantes por la utilización del término pararromaní (Matras 2011: 294).

Las variantes pararromaníes están documentadas en varias áreas periféricas de Europa (en Gran Bretaña, Escandinavia, en la Península Ibérica, en Grecia), donde sustituyeron completamente al romaní flexivo. Sus hablantes, por lo general, denominan estas variantes como "lenguas romaníes" debido a la presencia de léxico romaní. 


\subsection{Estructura lingüística del pararromaní}

Se entiende por el término pararromaní una lengua mixta cuyo léxico es generalmente romaní, mientras que la gramática (la fonología, la morfología y la sintaxis) proceden de la lengua mayoritaria (Bakker 1998: 71-72).

En los pararromaníes puede observarse una estricta línea divisoria entre la gramática y el léxico. Mientras que los morfemas ligados provienen siempre de la lengua de contacto, los morfemas gramaticales libres pueden proceder tanto de la lengua de contacto como del romaní, si bien es el romaní el que proporciona los morfemas léxicos libres, por ejemplo: no camel-o 'no quiero', pero también nasti camel-o. Las palabras con significado gramatical y léxico (por ejemplo, los pronombres) también pueden proceder del romaní y de la lengua de contacto (Boretzky - Igla 1994).

No se trata, por lo tanto, de un caso de mezcla de códigos dentro del discurso (code-switching) ni dentro de la frase (code-mixing), puesto que el límite entre ambas lenguas se halla en la palabra misma. Además, la mezcla de los códigos lingüísticos supone el conocimiento de ambos sistemas lingüísticos que se alternan en el discurso. Los hablantes que utilizan el pararromaní, sin embargo, suelen conocer solo el sistema gramatical de la lengua anfitriona, pero incluso el conocimiento del léxico romaní es limitado. Una característica común de los pararromaníes es también la aparición de formas no estándares que no admiten ninguno de los sistemas gramaticales.

Los subsistemas lingüísticos de los pararromaníes se rigen por determinadas normas generales. El subsistema fonológico se ha adaptado a la lengua mayoritaria, si bien la distribución de fonemas y su proporción en el texto se asemeja más bien al romaní (para la discusión sobre el caló, véase Bakker 1995: 130)

El subsistema gramatical procede, principalmente, de la lengua mayoritaria, aunque en ocasiones aparecen también residuos de palabras funcionales (pronombres, deixis de lugar, etc.) y de flexión romaní (principalmente nominal, la verbal se restringe mayoritariamente a la forma 3SG). En el subsistema léxico advertimos, además del elemento romaní y de contacto, la presencia de términos procedentes del argot y otros sociolectos marginales. Los diccionarios de pararromaníes incluyen generalmente unas 600 etimologías romaníes que contienen un léxico básico.

El pararromaní más antiguo y mejor documentado es el caló. La documentación actual de pararromaníes es más bien esporádica y acredita su repliegue. Véanse a continuación unos ejemplos del parraromaní español caló (elementos romaníes vienen en cursiva):

Caló (1): Ne chiá mangue con tusa. (Torrione 1988)

Español: No me voy contigo.

Caló (2): Las ducais me marelan. (Bright 1818: LXXXVII)

Español: Las penas me matan.

\subsection{Teorías de la emergencia de los pararromaníes}

Los pararromaníes emergieron independientes de sí mismos en diversos lugares. La génesis de las lenguas mixtas no ha sido claramente establecida hasta la fecha (Bakker 1995: 126; para la discusión más detallada, véase Matras 2011: 289-291). Una de las teorías supone que el pararromaní 
nació como consecuencia de la mezcla de la población romaní con grupos locales marginales. Las comunidades mixtas desarrollaron después su propio código emblemático. Esta variante mixta, a continuación, sustituyó el romaní flexivo como un símbolo de la identidad y el romaní fue abandonado. Este proceso se asimilaría a la génesis de las lenguas mixtas en comunidades con hogares mixtos (como, por ejemplo, michif), que se produce de forma relativamente rápida (Bakker 1998).

Boretzky (1983) señaló que el sustento del léxico romaní es incompatible con el proceso de disolución de la lengua y sugirió la creación como resultado del desplazamiento lingüístico, si bien el sustento del léxico romaní otorga a la lengua romaní un símbolo de identidad y de lengua críptica.

Según la mayoría de las teorías citadas sobre el nacimiento de los parraromaníes (Matras 2011; y Bakker - Van der Voort 1991), estas lenguas mixtas pueden ser el resultado de:

- Una regramaticalización basada en el préstamo masivo de la gramática de la lengua mayoritaria al romaní (Thomason - Kaufman 1988);

- La relexificación, basada en el préstamo masivo de léxico romaní a la lengua mayoritaria, si bien la pérdida de competencia en la gramática romaní se produce posteriormente;

- La pidginización y la consiguiente regramaticalización (el romaní en posición de lengua prohibida no podía cumplir todas las funciones de comunicación y se redujo progresivamente hasta que para expresarse fue necesario empezar a utilizar la gramática de la lengua de contacto);

- La creación intencionada de un nuevo idioma (intertwining), que supone el entrelazamiento consciente del léxico romaní con la gramática de la lengua mayoritaria, refiriéndose a la función del romaní como de lengua críptica (en una situación en la que está prohibido el uso del romaní para la comunicación, los hablantes del romaní crean una lengua híbrida cuyas palabras importantes sean romaníes, y por lo tanto inaccesibles a la mayoría de la población, pero que, sin embargo, a primera vista recordará un dialecto desconocido de la lengua de contacto. Sirva como testimonio de esta hipótesis principalmente el carácter críptico de estos dialectos mixtos. Sus hablantes son muy reacios a que su lenguaje sea revelado a cualquier persona que no pertenezca al grupo. Además de la función de lengua críptica, el romaní puede entenderse también como símbolo de identidad étnica;

- Desplazamiento lingüístico (language shift, cambio lingüístico) que cuenta con la pérdida de la competencia lingüística en romaní en las generaciones más jóvenes que han adoptado completamente la lengua de contacto y la consiguiente relexicalización, al producirse por influencia de las generaciones más viejas (o de la sociedad de romaníes con vida más tradicional) la inclusión de determinadas expresiones de romaní (Boretzky - Igla 1994).

En resumidas cuentas, según las opiniones actuales de lingüistas destacados que se dedican a la lengua romaní parece ser plausible que los pararromaníes fueran creados más o menos de forma consciente a fin de ocultar el contenido del discurso. Desde la perspectiva del romaní se produjo una regramaticalización, mientras que desde la perspectiva de la lengua de contacto se trató de una relexicalización.

El papel más importante viene dado por los siguientes factores: social (el estatus social de los hablantes de la lengua mixta, la mezcla de población, etc.), el bilingüismo, la diglosia, el olvido de 
una de las lenguas, el préstamo gramatical y lexical, la función comunicativa de la lengua mixta (por ejemplo, una lengua críptica).

A partir de un análisis de una muestra de DNA de los romaníes portugueses, Gusmão et al. (2008) confirman lo siguiente:

- El claro parentesco genético con el resto de romaníes en Europa demuestra su origen en la India, la permanencia durante un largo período de tiempo en el Oriente próximo y los Balcanes y la llegada a la Península Ibérica a través de Europa;

- El índice de parentesco genético con los ciudadanos de la Península Ibérica (españoles y portugueses) es elevado, lo que, en nuestra opinión, podría confirmar la hipótesis de la mezcla con grupos marginales de población defendida por Bakker.

\subsection{Historia lingüística de los romaníes en la Península Ibérica ${ }^{3}$}

La llegada de los romaníes a la Península Ibérica suele datarse a más tardar en la primera mitad del siglo XV (el primer documento claro e indiscutible es de 1425). Con su llegada se produjo un encuentro de sociedades con una identidad considerablemente distinta, de la que su diferente lengua constituía una parte importante. Probablemente y de forma rápida, los romaníes hicieron suya la lengua que necesitaban para la comunicación con la población del entorno. Con el paso del tiempo, como consecuencia del contacto con la población mayoritaria, se convirtieron en hablantes bilingües. Su lengua materna, sin embargo, era inicialmente sin duda el romaní flexivo que hablaban en la familia y en el grupo, los niños muy pequeños sabían seguramente solo romaní y con su progresiva independización aprendían la lengua mayoritaria. La documentación del romaní ibérico que se hablaba entre los siglos XV y XVII es muy escasa ${ }^{4}$.

El documento más antiguo sobre el romaní en el territorio de la actual España es un manuscrito datado hacia la mitad del siglo XVI, el Aucto del finamiento de Jacob (al parecer de origen sevillano) (Adiego 2013a), en el que aparece una pareja de personajes gitanos que hablan un romaní flexivo ${ }^{5}$ (entre ellos) y español con la inclusión frecuente de palabras romaníes (dirigidas a unos pastores). Las frases indicadas podrían considerarse un importante testimonio de bilingüismo del pueblo roma en el siglo XVI y un fenómeno de code switching y code mixing.

Bakker (1995: 141) constata que los rroma españoles seguramente perdieron muy pronto su lengua y que el romaní flexivo pudo ser sustituido por la lengua mixta caló unos 150 años después de su llegada, es decir, alrededor de $1600^{6}$, puesto que para el siglo XVIII el caló español ya tenía el carácter de una lengua mixta (cf. el Ms. 3929, de la Biblioteca Nacional de Madrid, transcrito con

3 Por motivos de espacio, nos limitamos aquí al caló español, dejando de lado de momento las historias lingüísticas de otros pararromaníes peninsulares, como el caló vasco (Bakker 1991) o el caló catalán (Ackerley 1914-1915; Escudero 2004; Escudero y Adiego 2001; o Adiego 2012).

4 Para las fuentes históricas del romaní en general, véase Adiego 2020.

5 Como apunta Adiego en su comentario sobre los pasajes romaníes del texto, hay que obrar con prudencia; el autor del texto estaba seguramente familiarizado con el romaní de la época, pero no está claro hasta qué punto podemos interpretar dichos pasajes como "romaní flexivo". 
errores por Hill 1921 y reestudiado magistralmente por Adiego 1998). La única documentación de restos del romaní flexivo se halla en el vocabulario español-gitano del Marqués de Sentmenat, editado y estudiado por Adiego (2002), pero en este caso se trata, de hecho, del caló catalán.

La lengua romaní, por supuesto, no disfrutaba de una posición de igualdad con respecto a las lenguas de alrededor. Era la lengua de una población minoritaria que a principios de 1499 estaba marginalizada y perseguida, una circunstancia que fue incrementándose al igual que en otras partes de Europa durante el siglo XVI y, principalmente, durante los siglos XVII y XVIII. La represión afectaba también a la lengua, cuyo uso fue prohibido. Se trataba, por tanto, de una situación de diglosia extrema.

La sustitución ya mencionada del caló y del argot tiene sus raíces en la marginalización de la etnia romaní y de su no diferenciación - ya intencionada o por desconocimiento - del resto de los grupos marginales de la sociedad. Por otro lado, el contacto entre el pueblo romaní y estos colectivos está ya acreditado en fuentes tempranas del caló, donde aparecen expresiones del argot, en concreto en el Guirigay de Jitanos (el Ms. 3929) (Adiego 1998). En el antiguo argot español (germanía), sin embargo, no encontramos ningunas expresiones procedentes claramente del romaní. A partir de esta influencia mutua asimétrica es posible inferir que los rroma, quizás, acogieran entre ellos a los repudiados sociales y, con ellos, su argot. Esta estrategia social, en el caso de que se produjera a mayor escala, podría ser también una de las causas de la desintegración del romaní flexivo7.

A pesar de que las circunstancias para la emergencia de los pararromaníes ibéricos continúan sin ser claras, en nuestra opinión, los factores ya mencionados que ejercían una influencia mutua desarrollaron un papel clave, al menos en el caso del caló: el bilingüismo prolongado, la marginalización de la etnia romaní y el contacto con otros grupos marginales, una sedentarización forzada, la asimilación y el rechazo de la lengua romaní en el periodo que se extendía del siglo XVI al XVIII y la moda consiguiente del flamenquismo en el siglo XIX.

\section{Recepción del caló en la sociedad española. Miradas desde la sociedad mayoritaria y desde la comunidad gitana ${ }^{8}$}

El caló ha sido en el español europeo un término polisémico en el que no siempre ha estado claro qué factores obedecen al curso de la historia y qué son proyecciones de estereotipos nacidos en el seno de la sociedad mayoritaria. Hoy en día el término suele designar 'la lengua de los gitanos españoles', sin embargo, en el pasado - y en algunas variedades geográficas del español hasta hoy día (cf. Lara 1992) - solía significar 'el argot de la delincuencia' o las dos cosas a la vez, sin trazarse una línea divisoria nítida.

A continuación, vamos a dar breve repaso por las acepciones del término para saber "de qué se ha hablado cuando se ha hablado del caló" a lo largo de la historia. Nos vamos a centrar

7 No obstante, como señala Adiego (2012) en su trabajo sobre el caló catalán en la obra literaria de Juli Vallmitjana, puede que la influencia no haya sido en realidad tan importante y que los hablantes hayan podido ser capaces de diferenciar qué proviene del argot y qué tiene el origen romaní, puesto que en la obra de Vallmitjana prácticamente no encuentra argotismos en el caló, pero sí hay gitanismos en el argot; los mismos resultados los ofrecen también las investigaciones de campo (Adiego 2005a y 2005b; Gamella, Fernández y Adiego 2015).

8 Este apartado está basado en gran parte en Buzek 2016a. 
básicamente en una mirada desde el exterior, para tratar la evolución de la percepción del caló como exónimo.

En esta parte del estudio mencionaremos algunas fuentes para el estudio del caló, sobre todos sus diccionarios (Adiego 2005c = 2006; Buzek 2011a; Krinková 2014 y 2015), para ver cómo los autores percibían y caracterizaban el caló, con qué actitudes pragmáticas y sobre todo ideológicas. En casos de obras del pasado no tenemos alternativas, puesto que son siempre miradas desde el punto de vista de la sociedad mayoritaria (Torrione 1993). En esta ocasión vamos a excluir de nuestras consideraciones la imagen del caló asociada a los repertorios del argot de la delincuencia, puesto que ya nos hemos dedicado a ellos en otras ocasiones (Buzek 2011b y 2016b).

La mayoría de los autores de los siglos XVIII, XIX y XX presentaban el caló como un código inventado, "como un argot del tiempo, próximo al habla alegórica de los ciegos y a la jerga germanesca de los ladrones, mendigos y rufianes" (Torrione 1993: 140), una imagen que pasaría a la experiencia lingüística compartida por los hispanohablantes a nivel general y también "allende los Pirineos como un registro de tercera categoría (Torrione 1993: 147). Además, a los gitanos se les negaba frecuentemente la etnicidad propia. Véanse algunos ejemplos:

[L]os que andan en España no son Gitanos, sino enxambres de Zánganos, y hombres ateos, y sin ley ni Religion alguna, Españoles que han introducido ésta vida, ò secta del Gitanismo, y que admiten à ella cada dia gente ociosa y rematada de toda España. [...] toda maldad hacen à su salvo, confiriendo entre sí en lenguage con que se entienden sin ser entendidos, que en España se llama GERIGONZA. (Sancho de Moncada 1779 [1619]: 204, 210)

[E]ste lenguage, llamado gerigonza por los españoles, se ha fingido probablemente por los gitanos de España para suplir por el nativo que habian olvidado, ó que habia perecido con la mezcla de los muchos españoles foragidos que se habian unido con ellos. En España, como tambien en Italia, los gitanos han olvidado y perdido casi totalmente su lenguage nativo, y queriendo continuar tratándose con un lenguage desconocido á los españoles e italianos, han inventado algunas palabras; y otras muchas las han formado, alterando la significacion de las italianas y españolas, ó invirtiendo sus sílabas (Hervás y Panduro 2008 [1800-1805]: 312).

La lengua propia de los gitanos no se descubre ni se puede descubrir en los que se esparcieron por los reinos occidentales de Europa, sino solamente en los que quedaron y aun hay en los orientales. Aquellos se esparcieron y recibieron en su compañía gran número de foragidos europeos, por lo que su idioma se adulteró fácilmente y pereció á poco tiempo. [...] En España como también en Italia, los gitanos han olvidado y perdido casi totalmente su idioma nativo, y queriendo continuar tratándose con un lenguage desconocido de estas dos naciones, han inventado algunas palabras y formado otras alterando su significación ó invirtiendo sus sílabas (Trujillo 1844: 15-16).

Hasta cierta época ha tenido mucha variacion su lenguaje, por las diferentes personas de varios reinos que se les agregaban, ya fugitivos, ya vagabundos, ociosos ó los que no encontraban medios de buscarse el sustento en su pais: [...]. En esta nacion [España] el último lenguaje que compusieron es del que se tratará, y aunque muchos creen que todos lo hablan, debe hacerse una advertencia de ello, pues los mas civilizados no lo entienden, y el que usan entre sí, y no con frecuencia, es sacado de aquel y del mal andaluz, con lo que componen una jerga semijocosa; asi como los que andan ambulantes por los campos y montes, que se ocupan de hacer canastas y esquilar, son los que más bien lo poseen, aunque no con mucha perfeccion (Jiménez 1997 [1853]: 6-7). 
El caló hablado por los gitanos no es tal como tiene su representación en los diferentes vocabularios que se han dado a luz. Además, son muy contados los individuos que lo dominan: los más civilizados apenas lo entienden, y el que usan entre sí se reduce, en los que más, a alguna que otra palabra procedente del caló primitivo, mezclada con el mal andaluz. Los que ambulan por los despoblados son los que mejor lo poseen, aunque con imperfección [...], todas las voces muy alteradas y corrompidas; formaciones caprichosas, sin reglas, fundamento ni razón [...]; con intromisión fraudulenta de voces de la jerga germanesca; en la cual también y a la par se ha ingerido el caló; de forma que éste resulta agermanado y la germanía aparece agitanada (Pabanó 2007 [1915]: 183-184).

Por la misma índole de su naturaleza y de sus costumbres, son los gitanos más afines a la sociedad delincuente que a la sociedad común; sin que esto quiera decir que tal afinidad haga que se confunda jamás la raza gitana con los hampones: sólo existe entre ellos cierta vecindad y cierta semejanza de inclinaciones (Pabanó 2007 [1915]: 186).

Han sido muy pocos los autores que no hayan incurrido en semejantes afirmaciones y que se hayan limitado a ofrecer una imagen más o menos fidedigna de la situación lingüística y sociolingüística del caló, es decir, una lengua mixta (un pararromaní). Los autores más representativos en este sentido serían José Antonio Conde, el autor del "Vocabulario de lengua ethigitana o de los gitanos", de comienzos del XIX (transcrito en Torrione 1988; cf. también Ruiz Fernández 2005; Adiego 2013a y 2013b) y George Borrow con su obra The Zincali (1843 [1841]) (Adiego y Martín 2006; Adiego 2008).

$[\mathrm{H}]$ aviendo con exacto examen registrado las derivaciones, $\mathrm{y}$ formaciones de nombres y verbos de la lengua Ethigitana, en nada substancial se diferencia de la nuestra (Conde, en Torrione 1988: 365); Los Ethigitanos forman los tiempos de sus verbos como nosotros de los nuestros, pues ya he dicho que en lo substancial corre todo parejo (Conde, en Torrione 1988: 368).

[Q] uando los Gitanos no se acuerdan de sus voces propias, ó en su lengua no hay termino que explique la cosa, entonces hablan assi, agitanizando" (Conde, en Torrione 1988: 380)

The Gypsy dialect is at present very much shattered and broken, being rather the fragments of the language which the Gypsies brought with them from the remote regions of the East than the language itself: it enables, however, in its actual state, the Gitanos to hold conversation amongst themselves, the import of which is quite dark and mysterious to those who are not of their race, or by some means have become acquainted with their vocabulary (Borrow 1843 [1841]: 315).

Though the words or a part of the words of the original tongue still remain, preserved by the memory amongst the Gitanos, its grammatical peculiarities have disappeared, the entire language having been modified and subjected to the rules of Spanish grammar, with which it now coincides in syntax, in the conjugation of verbs, and in the declension of nouns. Were it possible or necessary to collect all the relics of this speech, they would probably amount to four or five thousand words; [...] for it is necessary to state here, that though such an amount of words may still exist amongst the Gitanos in general, no single individual of their sect is in possession of one-third part thereof, nor indeed, we may add, those of any single city or province of Spain (Borrow 1843 [1841]: 332).

Si nos preguntamos por las razones y buscamos una interpretación contextualizada más amplia, podríamos leer las opiniones sobre el caló (y sobre los gitanos en general) como una manera 
de procurar resolver un inminente conflicto ideológico. De acuerdo con Van Dijk (1999: 32), "el conflicto ideológico sólo es posible entre culturas, mientras el conflicto ideológico dentro de las culturas necesita definirse en términos sociales, específicamente en los (sub)grupos determinados y sus creencias". Por tanto, si el caló fuera reconocido por autores españoles como una lengua (aunque mixta), propia de un grupo venido desde fuera, esto dañaría la imagen de España como un estado nacional (relativamente) homogéneo, una comunidad imaginada, en términos de Anderson (1991 [1983]), puesto que

[L]a lengua tiene mucho más que un sentido semiótico; tiene también un sentido retórico. La lengua no es entonces sólo un índice que apunta a una identidad colectiva, sino que también permite que las identidades colectivas emerjan y las naciones sean imaginadas (García 2007: 380).

Por ello autores españoles como Sancho de Moncada y Hervás y Panduro insistían tanto en que los gitanos de su época no eran una etnia independiente, sino unos simples vagabundos y criminales generalmente de origen nacional, aunque en principio corregibles y reintegrables al cuerpo del Estado, si se les aplicaban las mismas penas que a los vagabundos (Vázquez García 2009: 8384). Negando la etnicidad de los gitanos sería posible negar y borrar también los últimos restos de su lengua, presentarla como una jerga de los delincuentes (nacionales) e imaginar una comunidad homogénea, una nación española, en la que los gitanos no presentaban un conflicto ideológico (entre dos culturas distintas), sino "tan solo" un problema social dentro de la comunidad que se resolvería mediante la asimilación, y esta empezaría erradicando su lengua. Prueba de ello serían los comentarios de Jiménez y de Pabanó de que "los más civilizados apenas lo entienden [el caló]".

Para procurar contrarrestar el peso del exónimo, a continuación vamos a ofrecer una mirada desde el interior de la comunidad gitana española misma. Nos interesará ver cómo reaccionan los gitanos mismos hacia el caló, si se identifican con la voz y con todo lo que implica o qué otro término prefieren o preferirían, es decir, si la actitud exógena de la sociedad mayoritaria ha podido haber influido en la percepción y en el valor que se aprecia últimamente desde el prisma endógeno de la comunidad gitana en España. Para ello, nos basaremos sobre todo en trabajos de autores gitanos (Ramírez Heredia 1994; Jiménez Gabarri 1999; y Jiménez González 2009) y en las encuestas procedentes de recientes trabajos de investigación de campo (Adiego 2005a y 2005b; Gamella et al. 2015).

En primer lugar, es preciso advertir que hay un consenso general formulado desde el propio ámbito de la comunidad gitana, y es que "si todo lenguaje tiene como misión fundamental la comunicación, el caló hace tiempo que dejó de ser útil a este respecto" (Jiménez González 2009: 154). Asimismo, creemos que la mayoría de las personas implicadas (gitanas o no gitanas) suscribiría la definición del caló de Ramírez Heredia (1994: 181): "[h]abla de los gitanos españoles que usan algunas palabras del romanó y aplican en su integridad la gramática española".

En cuanto a la extensión del uso real cotidiano -activo y pasivo-, Gamella et al. (2015: 64) anotan que "our data most likely measures passive knowledge of this lexicon, because not much active use seems to be made of it". Los mismos datos los aporta también Adiego (2005a), aunque en otro estudio (Adiego 2005b: 6), con otro grupo de informantes (una familia gitana de Épila, de Aragón), apunta que: 
[T] hey were able to enunciate complete sentences in Caló with relative ease. This is very unusual: in general, the Gypsy informants I know are only able to remember several words, many times with difficulty, and cannot build entire sentences in a spontaneous way.

La actitud de los propios gitanos hacia el caló se perfila como bastante ambigua. Por una parte, autores próximos a las asociaciones culturales y ciudadanas gitanas (aquí representados por Ramírez Heredia y Jiménez Gabarri) a veces rechazan el concepto del caló por referirse a una lengua mixta, a un pararromaní, y no a una lengua independiente y propia en todos los planos. Dice por ejemplo Jiménez Gabarri (1999: 27) que:

[L]os gitanos de España hemos mezclado el romanó con el castellano, de forma que esto ha dado como resultado la corrupción del romanó original y que la castellanización del romanó nos impide a los gitanos españoles entendernos con los gitanos europeos.

Por tanto, desde el ámbito de las asociaciones gitanas ha surgido un impulso para reconstruir o, mejor dicho, reinventar un romaní ibérico, una variante estandarizada del romaní peninsular, conocida también bajo el nombre de Romanó-Kaló. Sin embargo, según comenta Jiménez González (2009: 157),

[A] pesar de sus esfuerzos por difundir sus hallazgos esta variante del caló estandarizado no ha trascendido más allá de algunos artículos publicados en Nevipens Romaní. Y no ha tenido aceptación tampoco entre los lingüistas romaníes.

Y prosigue Jiménez González (2009: 159) diciendo que "el intento de reintroducir el uso del romanó en España es una cuestión elitista y, por ello, minoritaria”. Otro tipo de rechazo del caló -aunque probablemente se tratará aquí más bien de palabras individuales de origen romaní que los gitanos ya no perciben como suyas, sino como términos del argot español-viene motivado por el hecho de que los gitanos identifican los últimos restos léxicos del pararromaní con el argot de la delincuencia. Por tanto, serían ellos mismos los que contribuyen al empobrecimiento del caló en su totalidad, puesto que, a nivel de una lengua mixta, el léxico es su elemento distintivo más destacado. Este patrón de conducta ha sido documentado en varias ocasiones por Gamella et al. (2015: 65), como podemos leer en el siguiente fragmento:

First, they argued that some of the proposed terms had become part of colloquial Spanish, and therefore were apayao or non-Gypsy. Secondly, several informants complained that some of the words in the questionnaire were not "Gitano" but quincalleras, talegueras or choriceras, that is, typical of tinkers, thieves or prisoners. This is congruent with the notion that, in the last two centuries, Caló had become associated with the Germanía, the jargon used in prisons and criminal underworlds that increasingly had incorporated Caló voices.

La misma actitud la documentó también Román Fernández (1995: 99) en su estudio del caló vallisoletano. 
Otros gitanos han rechazado el caló -en su totalidad o determinados términos que identifican con el argot- porque lo han identificado con registros desprestigiados del habla. Eso está relacionado con la actitud que acabamos de comentar en el anterior párrafo, pero se ha incorporado aquí también la noción de falta de civilización, de la barbarie, tal como hemos visto más arriba en casos de diccionarios de Jiménez (1997 [1853]: 6-7) y de Pabanó (2007 [1915]: 183).

Lastly, a few informants excused themselves saying they knew little of "that language" because it was representative of "old" Gitanos (antiguos or rancios - literally rancid) and not the "modern" Gitanos they considered themselves to be. In a telling case, a Gitano in his forties indicated his rejection of the interview by saying: "Why are you asking me this? I am already civilised..." He associated Caló with the stigmatisation suffered by many "typical" Gitanos (Gamella et al. 2015: 65).

Sin embargo, para otros gitanos el término caló tiene connotaciones positivas, aceptan el pararromaní como propio y su cualidad de código mixto lo esgrimen con dignidad, como una parte de su identidad. Afirma al respecto Jiménez González que "el caló es una creación colectiva de los gitanos españoles" y "hoy día el caló sirve, básicamente como herramienta identitaria básica. Es decir, sirve para identificarnos como gitanos ante otros gitanos” (Jiménez González 2009: 152 y 154). En otras palabras (Gamella et al. 2015: 87), "Caló serves mostly as an emblematic token of ethnic affirmation and resistance. Secondly, it is also used as a resource for enabling exclusive, in-group communication in the presence of outsiders"; pero no en el sentido de lengua secreta, ya que "for most of the people contacted, Caló is not a secret language anymore" (Gamella et al. 2015: 63). Sería interesante poder identificar algún patrón sociolingüístico que explicara las diferentes actitudes dentro de la comunidad gitana, pero son pocos los datos para poder sacar conclusiones al respecto.

Y finalmente apuntan Gamella et al. que el último valor del caló sería el afectivo, una manera de recordar a los antepasados: "[i]n this sense the use of Caló adds a sense of community to the interaction between Gitano people, often providing a sense of remembrance of dear ones no longer present" (2015: 87).

En general podemos decir que los gitanos españoles aceptan con cierta fatalidad y nostalgia la paulatina pérdida del caló, pero discrepan en cómo interpretarla y asumirla dentro de la conciencia colectiva del grupo. Los representantes del colectivo adoptan diversas posiciones que van desde la defensa del caló como una parte de su propia identidad hasta un feroz rechazo por considerarlo el principal obstáculo para la integración y aplauden el abandono de las prácticas lingüísticas que singularizan a la comunidad:

Most Gitano people recognise that Caló has practically vanished from their lives and is rarely used today. "Nothing is left"; "We do not speak it"; "All is lost" are expressions often repeated in interviews. There is a perception of loss that is generally regretted. Most Gitanos accept the death of Hispanoromani or Caló, as unavoidable. Some even view the disappearance of Caló as another element of the necessary modernisation experienced by the Gitano minority (Gamella et al. 2015: 88-89). 


\section{Proyectos de revitalización del gitano-español: ¿apuestas por el caló o por el neorromaní ibérico?}

En las últimas décadas del siglo XX y en los comienzos del siglo XXI ha vuelto a surgir el interés por la lengua y la cultura gitanas en España tanto entre las personas ajenas al colectivo como entre los gitanos españoles mismos. En lo que se refiere a la política lingüística del gitano-español, podemos notar dos corrientes distintas: una de ellas ha apostado por el caló, es decir, por el pararromaní, mientras que la otra ha propuesto reconstruir el romaní ibérico a base de otros dialectos de la lengua gitana que tienen más vitalidad hoy día.

En primer lugar, vamos a hablar sobre la corriente del neorromaní ibérico. Como ya hemos mencionado más arriba, su primer impulsor ha sido el antiguo eurodiputado socialista de origen gitano Juan de Dios Ramírez Heredia, autor de varias publicaciones sobre la lengua y la cultura gitanas, igual que de numerosos artículos de reivindicación lingüística, política y cultural del pueblo gitano en España. Junto con sus colaboradores ha intentado reconstruir el neorromaní ibérico a base de los dialectos europeos del gitano mejor conservados y ha ido publicando su gramática por entregas en la revista de la Unión Romaní I Tchatchipen (ahora O Tchatchipen) ${ }^{9}$, aunque es cierto que admite en él algunas particularidades de la morfología del caló, creando así una variedad "de compromiso" entre el caló y el neorromaní. Asimismo, es autor de un manual de conversación para principiantes (Ramírez Heredia 2001). No obstante, como ha apuntado Jiménez González (2009; cf. supra), sus actividades han tenido poca repercusión entre la propia comunidad gitana. El mismo Jiménez González ha participado en varios proyectos colectivos de implementación del neorromaní ibérico (Jiménez González, Plantón García, Valet, Ropero Núñez, Román Fernández 2004; y Jiménez González, Cañadas Ortega 2011), pero no estamos seguros hasta qué punto sus propias iniciativas hayan tenido más alcance. Aquí conviene matizar que el proyecto de Jiménez González y Cañadas Ortega (2011) está inspirado, y enmarcado, dentro del movimiento de los intelectuales romaníes a nivel internacional que promueven la creación de un romaní común estándar, menos idiosincrásico y más en contacto con otras comunidades rromá a nivel internacional

Otra corriente ha intentado incentivar el estudio, la recuperación y posible revitalización de la variante pararromaní, el caló. Desgraciadamente, ha adolecido de muchos defectos metodológicos y de mucho diletantismo, aunque probablemente llevados a cabo con buenas intenciones:

- Chipí Calí. Curso de introducción a la lengua gitana, de Julián De los Reyes Serrano y Rafael Fernández Suárez (1987a y 1987b), un documento mecanografiado e incompleto ${ }^{10}$, está basado en los diccionarios de los aficionados del siglo XIX y de comienzos del XX, sobre todo en el de Pabanó 2007 [1915], es decir, aquel autor que argumentaba que los gitanos y el caló casi coincidían con los delincuentes y con el sociolecto de estos;

- Los manuales y glosarios de José Antonio Plantón García (1993 y 2003) también están basados en las fuentes de los aficionados y de hecho solo vuelven a perpetuar los tópicos sobre la lengua gitana en España;

9 En los números 2, 3, 4, 8, 9, 10 y 12.

10 Guardado en la biblioteca del Centro Sociocultural Gitano-Andaluz en Granada. La versión A lleva la signatura R.516 y a la versión B le corresponde a su vez el número R.514. 
- Comunidad gitana: documento didáctico intercultural (1998), un documento anónimo, tiene los mismos fallos, es decir, está basado en los materiales del caló espurio de la Afición;

- Epítome de gramática gitana, de José Flores López, un documento mecanografiado sin fechar, pero originado probablemente en los años 80 o 90 del siglo $\mathrm{XX}^{11}$. Es un documento "ecléctico", en parte basado en las fuentes de la Afición, pero contiene también una parte léxica probablemente original y no documentada en otras fuentes. Desgraciadamente, no tenemos más información ni sobre el autor ni sobre los datos de la obra;

- Cursos del caló organizados por las asociaciones gitanas, como fueron, por ejemplo, los cursos promovidos por el Centro Socio-cultural Gitano-español en Granada en los años 2001 y 2002. Sus materiales de estudio presentaban los mismos defectos.

Desde el punto de vista teórico-metodológico seguramente han sido mucho más coherentes los proyectos que apostaban por la revitalización del neorromaní ibérico. Sin embargo, todos tenían el mismo punto débil que, al final, los ha llevado a todos al fracaso.

Por una parte, sus promotores han ensayado un plan de política lingüística, han hecho una planificación de corpus (han confeccionado un diccionario, una gramática y una ortografía normativos), pero han fallado en la planificación de estatus y en la planificación de la adquisición de la lengua. En otras palabras, han ideado una política lingüística, pero sin una planificación lingüística, es decir, sin ponerla en práctica con efectividad (Amorós Negre 2013: 87-91). Creemos que el punto débil de todas estas iniciativas ha sido su elitismo, puesto que probablemente han pasado prácticamente inadvertidos entre la población gitana en España y no han llegado a convencer a la población gitana sobre las ventajas de recuperar una lengua propia.

Si tuviéramos que escoger uno de los enfoques arriba referidos, optaríamos primero por la revitalización que enlazara la lengua de los antepasados de los gitanos de hoy, o sea el pararromaní, y eso por varias razones. La primera razón es de índole práctica: aprender una lengua totalmente nueva (neorromaní) sería para los gitanos más difícil que aprender el pararromaní utilizando la gramática española, generalmente conocida (aunque con vestigios de la flexión romaní) y un vocabulario romaní que algunos todavía tienen, al menos parcialmente, en la memoria. La segunda razón es la motivación para aprender un idioma nuevo. Como resulta de las actitudes de la comunidad gitana que habíamos mencionado anteriormente, algunos hablantes perciben el caló como un símbolo de su identidad romaní y muchas veces se arrepienten de su desaparición, aunque la consideran inevitable. Por eso, opinamos que los miembros de la comunidad gitana percibirían la revitalización del caló antiguo y tradicional como más natural, en comparación con el aprendizaje de un constructo lingüístico creado artificialmente como sería el neorromaní o el romanó-kaló que, además, toma por objetivo primordial el entendimiento con otros grupos romaníes en Europa, lo cual es evidentemente un afán loable, pero en una situación en la que la mayoría de los miembros de la comunidad gitana no viaja al extranjero, no asiste a conferencias europeas (las romaníes incluidas) y no lee la prensa extranjera (ya que muchos tienen problemas con terminar la escolaridad obligatoria), resulta un afán algo desligado de la realidad.

Con esto no queremos decir que los gitanos interesados no deberían tener la posibilidad de aprender un romaní flexivo, sea el neorromaní o alguno de los dialectos romaníes hablados en 
Europa. Estamos convencidos de que para la recuperación de la lengua gitana en España (en cualquiera de sus variedades), sería más viable y más práctico emprender una planificación lingüística graduada: recuperar primero el caló (español, catalán, vasco) y solo después emprender un segundo paso en camino de un neorromaní estandarizado.

La revitalización del gitano español sería sin duda un proceso a largo plazo. Partimos del criterio de que debería basarse en la cooperación de varios expertos (sobre todo, lingüistas) y activistas provenientes de las filas de la comunidad gitana.

La tarea principal de los activistas consistiría en discutir el proyecto de la revitalización con los miembros de la comunidad gitana, subrayando los aspectos positivos del caló (símbolo de identidad, lengua de los antepasados) y desarraigando los prejuicios (el caló no es una jerga de ladrones, el bilingüismo es una capacidad deseable), y en motivar a los gitanos a la colaboración activa (por ejemplo, a la participación en las investigaciones de campo).

La tarea de los lingüistas constaría de más pasos. En una primera fase sería necesario realizar amplias investigaciones de campo y explorar qué expresiones del caló recuerdan los hablantes de hoy, y eso en varios territorios. Algunas de las investigaciones han sido realizadas recientemente (Gamella et al. 2015), es posible apoyarse también en las investigaciones de fechas anteriores (Leigh 1998; Román Fernández 1995; McLane 1977; o Gordaliza Aparicio 2001, este solo hasta cierto punto). Asimismo, hay que utilizar los extensos materiales accesibles, sobre todo de carácter lexicográfico, compararlos entre sí, depurarlos apartando el grano de la paja. En esta sección algo se ha hecho ya (por ejemplo, Adiego 1998, 2002, 2005a, 2005b, 2005c = 2006, 2008, 2016; Buzek 2011a, 2016b; Fuentes Cañizares 2008; Krinková 2015, 2016), sin embargo, todavía queda mucho trabajo por hacer. En una segunda fase se construiría un diccionario y una gramática (con acentuación de las particularidades gramaticales del caló frente al español y los residuos de la flexión romaní. Al mismo tiempo, habría que reflexionar sobre el tratamiento de la variación del caló, considerando especialmente las diferencias locales. Sería oportuna la colaboración con gitanos de varias partes, sobre todo, con los que dominan parcialmente el caló. Y solo en una última fase se crearía un manual de aprendizaje y se empezaría con la enseñanza del caló. El primer objetivo debería ser el dominio pasivo del caló. Todo lo demás dependería de la actitud y de la motivación de los hablantes mismos.

\section{Conclusiones}

Los intentos revitalizadores llevados a cabo hasta hoy parecen más bien fracasados. Es muy probable que en caso de que no se emprendan otros pasos, dentro de poco los gitanos españoles no conocerán más de la lengua de sus antepasados que unos pocos préstamos del caló que han entrado en el español coloquial.

No pensamos, sin embargo, que todo esté irremediablemente perdido. Las demás lenguas minoritarias en la Península Ibérica empiezan a vivir un cierto auge revitalizador que, además, se ve estimulado y subvencionado por la UE. La lengua de los gitanos españoles se encuentra en una situación específica, ya que la mayoría de los miembros de esta comunidad está todavía marginalizada, resolviendo más bien problemas de índole económica y social que cuestiones relacionadas con la autoconciencia étnica. Por tanto, opinamos que resulta imprescindible la cooperación de 
los miembros de la comunidad gitana con los expertos entre los cuales prevalecerían, por lo menos en la primera etapa, los "payos", es decir, los no gitanos. Esta cooperación, a nuestro juicio, potencialmente fructífera, naturalmente no garantizará un éxito seguro, pero podría contribuir, al menos, a una amplia documentación lingüística del caló.

La revitalización es siempre un proyecto a largo plazo, sin embargo, el resultado del proyecto se revelaría solo en generaciones futuras y dependerá, de manera decisiva, de la participación activa de la población gitana misma. En este texto nos referíamos siempre, por motivos de espacio y de coherencia, al "caló español", es decir, el pararromaní originado por el contacto con el español, pero nuestras conclusiones se podrían extrapolar igualmente a otros calós ibéricos: el caló català, la erromintxela (el caló vasco) y el calão portugués.

\section{Referencias bibliográficas}

Ackerley, F. G. (1914-1915). The Romani speech of Catalonia. Journal of the Gypsy Lore Society, New Series, $8,99-140$.

Adiego, I.-X. (1998). The Spanish-Gypsy vocabulary of manuscript 3929, Biblioteca Nacional de Madrid (eighteenth century): A rereading. Journal of the Gypsy Lore Society, 5th series, 8, 1-18.

. (2002). Un Vocabulario español-gitano del Marqués de Sentmenat (1697-1762). Edición y estudio lingüístico. Barcelona: Edicions de la Universitat de Barcelona.

- (2005a). The Vestiges of Caló Today. In B. Schrammel, D. W. Halwachs, \& G. Ambrosch (Eds.), General and Applied Romani Linguistics. Proceedings from the $6^{\text {th }}$ International Conference on Romani Linguistics (pp. 60-78). Múnich: Lincom.

. (2005b). Recent fieldworks on Spanish Romani: lexical findings. Gypsy Lore Society. Annual Meeting. Granada, 2005-04-27. Inédito.

. (2005c). The first Caló dictionary ever published in Spain (Trujillo 1844): An analysis of its sources. Romani Studies, Series 5, 15, 2, 125-143.

. (2006). El primer diccionario kaló publicado en España (Trujillo, 1844): un análisis de sus fuentes. I Tchatchipen, 54, 23-36.

. (2008). When George Borrow met Spanish Romani. George Borrow Bulletin, 36, 15-28.

. (2012). Catalan Romani (caló català) in the work of Juli Vallmitjana: an initial appraisal. Zeitschrift für Katalanistik, 25, 305-320.

- (2013a). The oldest attestation of the Romani language in Spain: The Aucto del finamiento de Jacob (sixteenth century). Romani Studies, series 5, 23, 2, 245-255.

(2013b). La investigación sobre el caló español: algunas cuestiones teóricas y metodológicas. In N. Vila (Ed.), De parces y troncos. Nuevos enfoques sobre los argots hispánicos (pp. 17-34). Lleida: Edicions de la Universitat de Lleida.

. (2016). Un nonato diccionario gitano decimonónico: el Rotañulario dor Sersén al Calorró y de andalló al Sersén de Joaquín Simán (Pero Grullo). In I. Buzek (Ed.) Interacciones entre el caló y el español. Historia, relaciones y fuentes (pp. 77-98). Brno: Masarykova univerzita. 
Handbook on Romani Language and Linguistics (pp. 49-81). Cham: Palgrave-Macmillan.

Adiego, I.-X.; Martín, A. I. (2006). George Borrow, Luis de Usoz y sus respectivos vocabularios gitanos. Revista de Filología Española, LXXXVI, 1, 7-30.

Amorós Negre, C. (2013). Las lenguas en la sociedad. Madrid: Síntesis.

Anderson, B. (1991). Imagined communities: reflections on the origin and spread of nationalism. Ed. revisada y extendida. Londres: Verso.

Bakker, P. (1991). Basque Romani - a preliminary grammatical sketch of a mixed language. In P. Bakker \& M. Cortiade (Eds.), In the Margin of Romani. Gypsy Languages in Contact (pp. 56-90). Amsterdam: Publikaties van het Instituut voor Algemene Taalwetenschap.

. (1995). Notes on the genesis of Caló and other Iberian Para-Romani varieties. In Y. Matras (Ed.),

Romani in Contact. The History, Structure and Sociology of a Language (pp.125-150). Amsterdam-Philadelphia: John Benjamins.

- (1998): Para-Romani languages versus secret languages: Differences in origin, structure and use. In

Y. Matras (Ed.), The Romani Element in Non-Standard Speech (pp. 69-96). Wiesbaden: Harrassowitz Verlag.

Bakker, P.; \& Van der Voort, H. (1991). Para-Romani languages: an overview and some speculations on their genesis. In P. Bakker, \& M. Corthiade (Eds.), In the Margin of Romani. Gypsy Languages as Contact Languages (pp. 16-44). Amsterdam: Publikaties van het Instituut voor Algemene Taalwetenschap.

Bernal, J. M. F. (2016). Los gitanos en España y el possible origen del caló. In I. Buzek (Ed.) Interacciones entre el caló y el español. Historia, relaciones y fuentes (pp. 11-27). Brno: Masarykova univerzita.

Boretzky, N. (1983). Kreolsprachen, Substrate und Sprachwandel. Wiesbaden: Harraschovitz.

Boretzky, N.; \& Igla, B. (1994). Romani Mixed Dialects. In P. Bakker, \& M. Mous (Eds.), Mixed Languages. 15 Case Studies in Language Intertwining (pp. 35-68). Amsterdam: IFOTT.

Borrow, G. (1843 [1841]). The Zincali; or an account of the Gypsies of Spain. With an original collection of their songs and poetry, and a copious dictionary of their language. $3^{\mathrm{a}} \mathrm{ed}$. Londres: John Murray.

Bright, R. (1818). Travels from Vienna to Lower Hungary with Some Remarks on the State of Vienna during the Congress, in the Year 1814. Edimburgo: Archibald Constable and comp.

Buzek, I. (2011a). Historia crítica de la lexicografía gitano-española. Brno: Masarykova univerzita.

- (2011b). La noción de argot en la tradición lingüística hispánica y su primer diccionario: el Diccionario del argot español de Luis Besses (1905). In A. Butašová, et al. (Eds), Philologica XXI, Supplementum II (pp. 57-66). Bratislava: Univerzita Komenského Bratislava.

- (2016a). ¿Qué quiere decir caló? Matices de un glotónimo. Estudios de lingüística del español, 37, 263-283.

(2016b). Los estudios criminológicos y materiales internos de las fuerzas represivas del Estado español como fuentes para el estudio de gitanismos en el argot de la delincuencia durante los siglos XIX y XX. In I. Buzek (Ed.) Interacciones entre el caló y el español. Historia, relaciones y fuentes (pp. 99-111). Brno: Masarykova univerzita.

De los Reyes Serrano, J.; \& Fernández Suárez, R. (1987a). Chipi Calí. Curso de introducción a la lengua gitana. Curso para la integración gitana. Inédito [Ayuntamiento de Jerez, Delegación de Educación y Cultura].

- 1987b. Chipí Calí. Curso de introducción a la lengua gitana. Curso para la integración gitana. Inédito [Ayuntamiento de Jerez, Delegación de Educación y Cultura].

Escudero, J. P. (2004). Les gitans catalans et leur langue. Perpignan: Editions de la Tour Gile.

Escudero, J. P.; Adiego, I.-X. (2001). Vocabulari del kalò català. I tchatchipen, 33, 4-30. 
Flores López, J. (s/a). Epítome de gramática gitana. Inédito.

Fuentes Cañizares, J. (2008). Apuntes sobre el caló en la obra de George Borrow. Madrid: Vision Libros.

Gamella, J. F.; Fernández, C.; \& Adiego, I.-X. (2015). The long agony of Hispanoromani: The remains of Caló in the speech of Spanish Gitanos. Romani Studies, 25, 1, 53-93.

García, O. (2007). Lenguas e identidades en mundos hispanohablantes: desde una posición plurilingüe y minoritaria. In M. Lacorte (Ed.), Lingüística aplicada del español (pp. 377-405). Madrid: Arco/Libros.

Gómez Alfaro, A. (2009). Legislación histórica dedicada a los gitanos. Sevilla: Junta de Andalucía.

Gordaliza Aparicio, F. R. (2001). La lengua gitana en Palencia: restos y orígenes. Publicaciones de la Institución Tello Téllez de Meneses, 72, 207-249.

Gusmão, M. et al. (2008). A Perspective on the History of the Iberian Gypsies Provided by Phylogeographic Analysis of Y-Chromosome Lineages. Annals of Human Genetics, 72, 2, 215-227.

Hervás y Panduro, L. (2008). Catálogo de las lenguas de las naciones conocidas, y numeración, división, y clases de éstas según la diversidad de sus idiomas y dialectos. 6 vols. Alicante-Madrid: Biblioteca Virtual Miguel de Cervantes-Biblioteca Nacional. <http://www.cervantesvirtual.com/buscador/?q=Cat\%C3\%A1 logo + de+las+lenguas+de+las+naciones+conocidas $\% 2 \mathrm{C}+\mathrm{y}+$ numeraci $\% \mathrm{C} 3 \% \mathrm{~B} 3 \mathrm{n} \% 2 \mathrm{C}+\mathrm{divisi} \% \mathrm{C} 3 \% \mathrm{~B}-$ $3 \mathrm{n} \% 2 \mathrm{C}+\mathrm{y}+$ clases $+\mathrm{de}+\% \mathrm{C} 3 \% \mathrm{~A} 9 \mathrm{stas}+\mathrm{seg} \% \mathrm{C} 3 \% \mathrm{BAn}+\mathrm{la}+$ diversidad $+\mathrm{de}+$ sus + idiomas $+\mathrm{y}+$ dialectos\& $=0 \& \mathrm{y}=0 \& \mathrm{f}[\mathrm{cg}]=1>$

Jiménez, A. (1997). Vocabulario del dialecto jitano, con cerca de 3000 palabras y una relacion esacta del carácter, procedencia, usos, costumbres, modo de vivir de esta jente en la mayor parte de las provincias de España, celebridad en las fiestas, nombres y apellidos mas usuales, fisionomia y cuantos antecedentes se pueden tener de ellos, con varios rezos, cuentos, fábulas, versos, brindis, parte de la doctrina cristiana y ordenanza militar. Valencia: Librería París-Valencia [Ed. facsimilar de la 2a ed., Sevilla, Imprenta del Conciliador, 1853].

Jiménez Gabarri, A. (1999). Amarí shib romaní (Nuestra lengua gitana). s/n: J. Martínez.

Jiménez González, N. (2009). ¿El romanó, el caló, el romanó-kaló o el gitañol? Cincuenta y tres notas sociolingüísticas en torno a los gitanos españoles. Anales de Historia Contemporánea, 25, 149-161.

Jiménez González, N.; \& Cañadas Ortega, A. (2011). Sar san? ¿Cómo estás? Rromani ćhibăqo kùrso. Curso de Romanó. Madrid: Fundación Instituto de Cultura Gitana.

Jiménez González, N.; Plantón García, J. A.; Valet, J.; Ropero Núñez, M.; Román Fernández, M. (2004). Manual de Lengua Romaní. Málaga: Unidad de Recursos Europeos/Diputación Provincial de Málaga.

Krinková, Z. (2014). Prameny ke studiu caló a dalších iberských pararomštin. Romano džaniben, 21, 1, 91110.

. (2015). From Iberian Romani to Pararromani Varieties. Praga: Karolinum.

(2016). El vocabulario romanó documentado en los diccionarios del caló. In I. Buzek (Ed.) Interacciones entre el caló y el español. Historia, relaciones y fuentes (pp. 29-44). Brno: Masarykova univerzita.

Lara, L. F. (1992). El caló revisitado. In E. Luna Traill (Ed.), Scripta Philologica in Honorem Juan M. Lope Blanch a los 40 años de docencia en la UNAM y a los 65 años de vida. Lingüística española e iberoamericana, vol. 2 (pp. 567-592). México: Universidad Nacional Autónoma de México, Instituto de Investigaciones Filológicas.

Leigh, K. (1998). Romani elements in present-day Caló. In The Romani Element in Non-Standard Speech (pp. 241-282). Wiesbaden: Harrassowitz Verlag.

Matras, Y. (2011). Language Contact. New York: Cambridge University Press.

McLane, M. F. (1977). The Caló of Guadix: A Surviving Romani Lexicon. Anthropological Linguistics, 19, 7 , 303-319. 
Moncada, S. de. (1779). Espulsion de los gitanos. In Romances de Germanía de varios autores, con el vocabulario por la orden del a. b. c. para declaracion de sus términos y lengua. Compuesto por Juan Hidalgo: El discurso de la expulsion de los gitanos, que escribió Doctor Don Sancho de Moncada, catedratico de Sagrada Escritura en la Universidad de Toledo, y los romances de la germanía que escribió Don Francisco de Quevedo (pp. 201-222). Madrid: Don Antonio de Sancha.

Pabanó, F. M. [Manzano López, Félix]. (2007). Historia y costumbres de los gitanos. Colección de cuentos viejos y nuevos, dichos y timos graciosos, maldiciones y refranes netamente gitanos. Diccionario español-gitano-germanesco. Dialecto de los gitanos. Mairena de Aljarafe: Extramuros [Ed. facsimilar de la de Barcelona, Montaner y Simón, 1915].

Plantón García, J. A. (1993). Aproximación al Caló. Chipí Cayí. Albolote: Junta de Andalucía. Consejería de Asuntos Sociales.

- (2003). Los gitanos. Su cultura y su lengua. Proyecto Romani in Europe. Málaga: Unidad de Recursos Europeos/Diputación Provincial de Málaga.

Ramírez Heredia, J. de Dios. (1994). Cartas del pueblo gitano. Barcelona: Instituto Romanó de Servicios Sociales y Culturales.

- (2001). Primer manual de conversación en romanò-kalo - Angluno pustik pa vakeripen romanò-kalo. Barcelona: Instituto Romanó de Servicios Sociales y Culturales.

Román Fernández, M. (1995). Aportaciones a los estudios sobre el caló en España. Valencia: Universidad de Valencia.

Ruiz Fernández, C. (2005). La lengua gitana a finales del s. XVIII. In L. Santos Ríos (Ed.), Palabras, norma, discurso: en memoria de Fernando Lázaro Carreter (pp. 1055-1064). Salamanca: Ediciones Universidad de Salamanca.

Thomas, Sarag. G.; \& Kaufman, T. (1988). Language Contact, creolization and genetic linguistics. Berkeley: University of California Press.

Torrione, M. (1988). Del dialecto caló y sus usuarios: la minoría gitana de España. Tesis doctoral inédita. Perpignan: Université de Perpignan.

- (1993). La lengua del gitano de España, seña de identidad excluyente (siglos XV-XIX). In M. Torrione (Ed.), Lengua, libertad vigilada (pp. 129-153). Toulouse: Université de Toulouse.

Trujillo, E. (1844). Vocabulario del dialecto gitano. Madrid: Imprenta de D. Enrique Trujillo.

Van Dijk, T. A. (1999). ¿Un estudio lingüístico de la ideología? In G. Parodi Sweis (Ed.), Discurso, Cognición y Educación. Ensayos en Honor a Luis A. Gómez Macker (pp. 27-42). Valparaíso: Ediciones de la Universidad Católica de Valparaíso.

Vázquez García, F. (2009). La invención del racismo. Nacimiento de la biopolítica en España, 1600-1900. Madrid: Akal.

VV.AA. (1998). Comunidad gitana: documento didáctico intercultural. Sevilla: Junta de Andalucía. Consejería de Asuntos Sociales. 
